\title{
Cuckoo Search Algorithm using Lèvy Flight: A Review
}

\author{
Sangita Roy \\ ECE Department, Narula Institute of Technology, WBUT, Agarpara, K olkata, India. \\ roysangita@gmail.com \\ Sheli Sinha Chaudhuri \\ Electronics \& Telecommunication Engineering Department, Jadavpur University, Kolkata, India. \\ shelism@rediffmail.com
}

\begin{abstract}
Cuckoo Search (CS) is a new met heuristic algorithm. It is being used for solving optimization problem. It was developed in 2009 by Xin- She Yang and Susah Deb. Uniqueness of this algorithm is the obligatory brood parasitism behavior of some cuckoo species along with the Levy Flight behavior of some birds and fruit flies. Cuckoo Hashing to Modified CS have also been discussed in this paper. CS is also validated using some test functions. After that CS performance is compared with those of GAs and PSO. It has been shown that CS is superior with respect to GAs and PSO. At last, the effect of the experimental results are discussed and proposed for future research.
\end{abstract}

Index terms - Cuckoo search, Levy Flight, Obligatory brood parasitism, NP-hard problem, Markov Chain, Hill climbing, Heavy-tailed algorithm.

\section{INTRODUCTION}

At present, problem solving optimization algorithms are met heuristics and all of them are nature inspired. As soon as they are emanated, they are accepted by researchers and applied widely. Ant Algorithms were inspired from the behavior of ants in the wild. Particle Swarm Optimizations (PSO) was stimulated from the world of fish and bird, whereas the Firefly Algorithm was influenced by the flashing patterns of tropical fireflies. All of these bio-inspired met heuristic algorithms are being applied in problem solving optimization such as NP-Hard problems: the Travelling Sales Man Problem (TSP). The strength of all modern met heuristic algorithms are the fact that they mimic the best properties in nature, particularly biological systems emerged from natural selection over millions of years, of them two important features can be simulated numerically or algorithmically into two major features : intensification, and diversification. Intensification tries to search around the current best solutions and select the best candidates or solutions, whereas diversification utilizes the search space of the algorithm efficiently. CS algorithm revolves around the behavior of obligatory brood parasitism of some species of cuckoo as well as the Levy Flights (after the name of French mathematician Paul Pierre Levy) of some birds and fruit flies which follow the random walk of heavy tailed probability distribution step size. These observations are formulated into algorithms and implemented as a novel and new idea. CS is compared with other already existing popular optimization algorithms in connection with numerous optimization problems.

Cuckoo Hashing is another computing technique for resolving hash collisions of values in hash functions in a table. It derives the concept from some cuckoo species chicks pushing out of the nests other eggs or chicks at the time of hatching. The idea was derived from Ramses Pugh and Fleming Fiche Rodler in 2001. Since hybridization is possible between the above mentioned algorithms, as a result we can say that CS is derived from Swarm Intelligence. By statistical analysis it has been established that problem solving success of CS is far superior to PSO (PSO2007) [1]. Modified CS (MCS) is also made by Walton et al [2]. It delivers high convergence rate which out performs other optimizers. Basically it performs massively at high dimension bench mark objective functions. Consequently it can be applied on engineering problems. To be precise, the modification stresses on the information exchange between two best eggs or best solutions.

The paper is mainly organized according to the paragraphs as: i) Study of cuckoo behavior and Levy flight, ii) The primary concept behind the Cuckoo search with pseudo code, iii) Realization and mathematical results using different test functions, and iv)Comparative study between PSO and GA. Finally in conclusion it has been shown that less number of parameters (two in the given study) of the CS algorithm leads to its better efficiency. In future more complex parametric study can be carried out with more promising results in terms of the aim of speed of convergence. As a result of this the computational cost reduces.

\section{OBSERVATION ON CUCKOO BEHAVIOR AND LÈVY FLIGHTS}

A. Obligatory Brood Parasitism of Cucko 
The first impressions about cuckoos are their beautiful tone. But apart from their sound, they have a spectacular intrusion reproductive strategy. This is known as brood parasitism. There are two types of brood parasitism: non-obligatory and obligatory. In nonobligatory brood parasitism, cuckoo lays eggs in the nest of conspecifics (i.e., same species) and in their own nests as well. Examples: Bank Swallows, African Weavers. In obligatory brood parasitism cuckoos lay eggs in the nest of hetero-specifies, and do not require building the nest of their own and incubate the eggs. Examples: Brown- Headed Cowbirds and European Cuckoos. It has been reported that $1 \%$ of all bird species follow obligatory brood parasitism. Example: all African Honey Guides, almost half of the species of cuckoos, the Black Headed Duck in South America, Shiny Cowbirds, Screaming Cowbirds, Bronze Cowbirds and Giant Cowbirds. There are three types of brood parasitism: intraspecific brood parasitism, cooperative breeding, and nest takeover. Sometimes the host birds engage in fight with intruder cuckoos. Either the host birds may throw out the eggs if they find the eggs of invaders or may leave the nest and rebuild the nest elsewhere. The female cuckoos of the New World brood parasitic Tapera who are expert to imitate the colour and shape of the eggs of chosen host species, as a result of which, the probability of their eggs to be relinquished declines and intensifies their reproductively. The time of egg-laying of some parasitic cuckoo is also astonishing. Parasitic cuckoo lays eggs on the nest of host birds. Host bird incubates both the eggs of its own as well as the eggs of parasitic cuckoos. It is also amazing that the parasitic eggs hatch slightly earlier than that of the host eggs. Host birds incubate parasitic chicks as their own. By natural instinct, cuckoo chicks try to evict the host eggs randomly out of the nest for the sake of its own existence and food. By investigation, it has been found that cuckoo chicken imitate the sound of host chicken to obtain more food from the host mother.

\section{B. Concept of Levy Flights}

A Levy Flight can be thought of as a random walk where the step size has a Levy tailed probability distribution. The name Levy Flight came after the French mathematician Paul Pierre Levy. The term Levy Flight was coined by Benoit Mandelbrot who used specific definition of the distribution of the step sizes. Eventually Levy Flight term has been using to refer discrete grid rather than continuous space. It is a Markov Process. Exponential property of Levy Flight gives it a scale invariant property and they are used to model data for exhibiting/ showing clusters. In nature many animals and insects follow the properties of Levy Flight. Recent studies of Reynolds and Frye demonstrate that fruit flies or drosophila melanogaster covers the skies by using numerous series of straight flight paths/ routes followed by a sudden right angle turn which is a Levy-flight-style intermittent scale free search pattern. Hunter-gatherer forage pattern exhibit the typical feathers of Levy Flight, observed by Ju/'bonsai on human behavior. Studies also show that light rays follow Levy Flights in optical material [3]. Ultimately, it is being used in optimization search and significant results are emerging.

\section{THE BASIC IDEA BEHIND CUCKOO SEARCH}

Cuckoo Search (CS) is a new met heuristic search algorithm. It is characterized by three laws: each cuckoo lays one egg at a time and disposes the egg at a randomly chosen host nest, secondly the best nest with excellent quality of eggs will carry over to the next generation, and lastly the number of host nest is fixed and the probability of parasitic egg-discovery by the host bird is $\mathrm{p}_{\mathrm{a}} \in[0,1]$. The host bird can either throw out the parasitic egg or forfeit that nest for a better new nest. For practicality, in the later hypothesis it can be assumed that fraction $\mathrm{p}_{\mathrm{a}}$ of the nests are substituted by new nests (with new random solutions).In one form of fitness the quality or fitness of a solution is proposed to be proportional to the value of the objective function in case of a maximization problem. Other form of fitness can be proposed in the same manner as that of fitness function of GAs. In a simple manner of representation, each egg in a nest is represented by a solution and each cuckoo egg symbolizes a new solution. The aim is to exploit the new and probably finer/ superior solutions (cuckoos) to substitute a not-so good solution in the nests. More intricate cases like multiple eggs in a nest, symbolizing a set of solution, can be incorporated in this algorithm. Here the simplest approach, i.e., one egg in one nest, has been applied. Guided by the above three rules, the CS is abridged by the pseudo code:

\section{Cuckoo Search via Le'vy Flights}

\section{begin}

Objective function $\mathrm{f}(\mathrm{x}), \mathrm{x}=\left(\mathrm{x}_{1}, \mathrm{x}_{2}, \ldots \ldots \ldots \mathrm{x}_{\mathrm{d}}\right)^{\mathrm{r}}$

Generate initial population of

A host nests $x_{i}(i=1,2,3 \ldots \ldots, n)$

While ( $1<$ Maxgeneration) or (stop criterion )

Get a cuckoo randomly by Levy Flights

Evaluate its quality/fitness $F_{i}$

Choose a nest among $n$ (say, $\mathrm{j}$ ) randomly

if $\left(F_{i}>F_{j}\right)$,

Replace $\mathrm{j}$ by the new solution;

end

A function $\left(\mathrm{p}_{\mathrm{a}}\right)$ of worse nests

Are abandoned and new ones are built;

Keep the best solutions

(or nests with quality solutions);

end while

Rank the solutions and find the current best end
Postprocess results and visualization 
A new solution is symbolized by $\mathrm{x}^{(\mathrm{t}+1)}$ and a cuckoo is represented by $i$, then a Levy Flight is carried out by

$x_{i}^{(t+1)}=x_{i}^{(t)}+\alpha \oplus L e^{\prime} v y(\lambda)$,

Where $\alpha>0$ is the step size which is adjusted according to the scale of the problem of interests. In most of the cases $\alpha$ is assumed to be unity. Equation 1 represents the stochastic equation of random walk. Generally random walk is charecterised by Markov chain where the next state is represented by the present location (the first term of the above equation) and the transition probability (the second term). EX-OR symbolizes exclusive OR i.e., entry wise multiplication. This entry wise multiplication is equivalent as that of PSO, except that the random walk via Levy Flight is more efficient in exploring / acquiring the search space because its much longer step size increases exponentially in the last stage. Levy Flight is a random walk with the random step size following a Levy distribution.

Le'vy $\sim u=l^{-\lambda}, \quad(1<\lambda \leq 3)$,

Levy distribution has infinite variance with infinite mean with a power-law step size of a heavy tail as it is shown in fig 1.

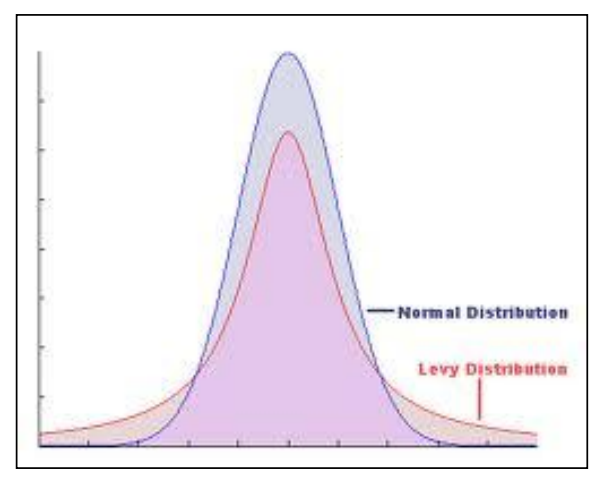

Figure 1. Levy and Normal Distribution [4]

Using Levy walk, few new solutions must be explored for the speed up of local search from the best solutions so far. It should be kept in mind as a warning that the system must not be trapped in a local optimum. For this reason, a portion or adequate amount of fraction of the new solutions must be cropped up from far field randomization with locations far enough from the current best solution. At a glance, both CS and hillclimbing in combination with few large scale randomizations appeared to be similar, but there is some salient dissimilarity. Firstly, like GAs and PSO, CS is a population based algorithm, at the same time it explores some kind of elitism and/or selection as that of harmony search. Secondly, the randomization is more efficient as the step size is heavy tailed with any possible large step size. Thirdly, the number of tuning parameters is less than GAs and PSO which in turn promises to be acceptable to a wider class of optimization problems. Moreover, an individual nest representing a solution makes CS adaptable to the class of Meta population algorithms.

\section{REALISATION AND NUMERICAL VERIFICATION}

\section{A. Authentication and Specification Studies}

After implementation, the algorithm has to be verified using test functions with analytical or known solutions. In this case, the bi-variate Michaelwicz function is used.

$$
f(x, y)=-\sin (x) \sin ^{2 m}\left(\frac{x^{2}}{\pi}\right)-\sin (y) \sin ^{2 m}\left(\frac{2 y^{2}}{\pi}\right)
$$

Where $m=10$ and $(x, y) €[0,5] \times[0,5]$ with global minima $f * \approx \approx-1.8013$ at $(2.20319,1.57049)$.

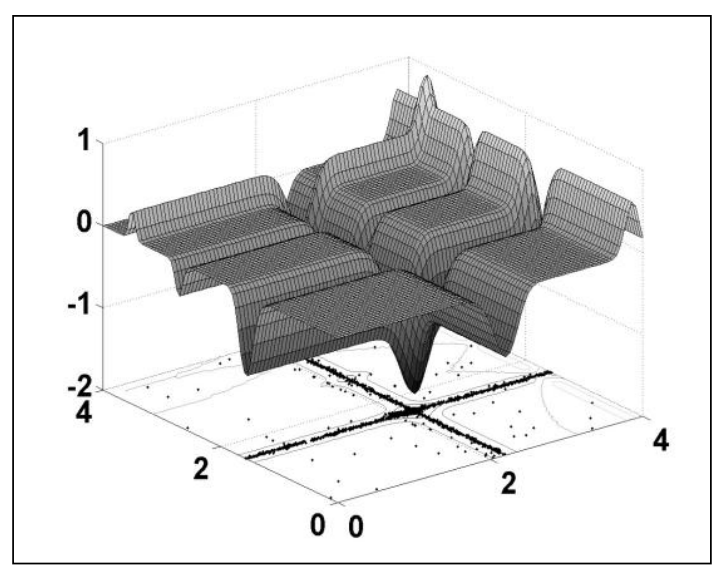

Figure 2. The landscape of bi-variate Michaelwicz Function [5]

The landscape of this function is shown in fig 2. CS finds these global optima easily and it is shown in fig. 3. With final locations of the nests marked $\diamond$. Here, the number of nests, step size and probability of parasitic egg-discovery used are $n=15, \alpha=1$ and $\mathrm{pa}=0.25$. In most of the simulation cases $n=15$ to 50 . The results from the figure show that most of the nests accumulate towards the global optimum as the optimum is approaching. In case of multimodal functions, the nests are scattered at different local optima. From the above discussion, it is clear if the numbers of nests are higher than that of the number of local optima, the CS scans all the optima simultaneously. In case of multimodal and multiobjective optimization problems, this advantage may be more pronounced. In this particular case, the number of host nests (i.e., the population size $n$ ) and the probability $\mathrm{p}_{\mathrm{a}}$ are varied where $\mathrm{n}=5,10,15,20,50,100,150,250,500$ and $\mathrm{p}_{\mathrm{a}}=$ $0,0.01,0.05,0.1,0.15,0.2,0.25,0.4,0.5$.Simulation result shows that $\mathrm{n}=15$ and $\mathrm{p}_{\mathrm{a}}=0.25$ are adequate for majority of the optimization problems. From the results and analysis, it has been deduced that the rate of convergence mostly is independent of the parameters used, which in turn leads to inessentiality of fine adjustment for any problems. Therefore, throughout the given problem, $\mathrm{n}=15$ and $\mathrm{p}_{\mathrm{a}}=0.25$ has been used. 


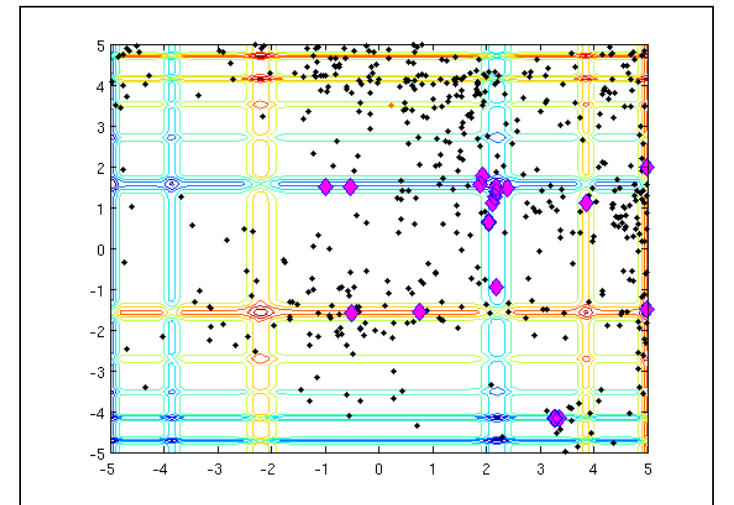

Figure 3. Search paths of nests in Cuckoo Search where the final location of the nests are denoted by $\diamond$ in the figure [6]

\section{B. Test Functions}

There are numerous benchmark test functions to verify the performance of optimization algorithms. All new optimization algorithms must be validated and tested against these benchmark functions. In this algorithm (CS), few test functions have been utilized for simulations.

1) Sphere Function-De Jong's first function

$f(x)=\sum_{i=1}^{d} x_{i}^{2}, \quad x_{i} \in[-5,12,5,12]$,

Where global minimum $\mathrm{f}_{*}=0$ at $\mathrm{x}_{*}=(0,0,-----, 0)$ with $\mathrm{d}$ dimension.

2) Easom's Test Function is unimodal

$f(x, y)=-\cos (x) \cos (y) \exp \left[-(x-\pi)^{2}-(y-\right.$ $\left.\pi)^{2}\right]$,

Where

$(x, y) \in[-100,100] x[-100,100]$,

With global minimum of $f_{*}=-1$ at $(\Pi, \Pi)$ in a very small region.

3) Shubert's Bivariate Function

$f(x, y)$

$-\sum_{i=1}^{5} i \cos [(i+1) x+1] \sum_{i=1}^{5} \cos [(i+1) y$

$+1)]$

Where 18 global minimas exists in the region $(\mathrm{x}, \mathrm{y}) \in[-$ $10,10] \times[-10,10]$. The global minimas have a value of -186.7309 .

4) Griewangh's Test Function

This function has many local minimas.

$$
f(x)=\frac{1}{4000} \sum_{i=1}^{d} x_{i}^{2}-\prod_{i=1}^{d} \cos \left(\frac{x_{i}}{\sqrt{i}}\right)
$$

Where a single global minima $\mathrm{f}_{*}=0$ at $\mathrm{X} *=(0,0,-----, 0)$ for all $-600 \leq x_{i} \leq 600$ where $i=1,2,---, d$.

5) Ackley's Function is multimodal

$$
\begin{aligned}
f(x)=-20 \exp \left[-0.2 \sqrt{\left(\frac{1}{\mathrm{~d}} \sum_{\mathrm{i}=1}^{\mathrm{d}} \mathrm{x}_{\mathrm{i}}^{2}\right)}\right] \\
\\
-\exp \left[\frac{1}{d} \sum_{i=1}^{d} \cos \left(2 \pi x_{i}\right)\right] \\
+(20+e),
\end{aligned}
$$

Where a global minima $\mathrm{f}_{*}=0$ at $\mathrm{x}_{*}=(0,0,---, 0)$ between $-32.768 \leq \mathrm{x}_{\mathrm{i}} \leq 32.768$ with $\mathrm{i}=1,2,----, \mathrm{d}$.

6) Generalized Rosen rock's Function

$$
\begin{aligned}
f(x)=\sum_{i=1}^{d-1}\left[\left(1-x_{i}\right)^{2}\right. \\
\\
+100\left(x_{i+1}\right. \\
\left.\left.-x_{i}^{2}\right)^{2}\right]
\end{aligned}
$$

Where a minima $\mathrm{f}\left(\mathrm{x}_{*}\right)=0$ at $\mathrm{x}_{*}=(1,1,---, 1)$.

7) Sahwefel's Test Function is multimodal

$f(x) \sum_{i=1}^{d}\left[-x_{i} \sin \left(\sqrt{\left|x_{i}\right|}\right)\right],-500 \leq x_{i} \leq 500$,

Where a global minima $f_{*}=-418.9829 d$ at $x_{i}{ }^{*}=420.9687$ with $\mathrm{i}=1,2$, ----d.

8) Rastrigin's Test Function

$$
f(x)+10 d+\sum_{i=1}^{d}\left[x_{i}^{2}--10 \cos \left(2 \pi x_{i}\right)\right],
$$

Where a global minima $\mathrm{f}_{*}=0$ at $\mathrm{x}_{*}=(0,0,---0)$ in a hypercube $-5.12 \leq \mathrm{x}_{\mathrm{i}} \leq 5.12$ with $\mathrm{i}=1,2,---, \mathrm{d}$.

9) Michalewicz's Test Function It has d! local optima

$f(x)=-\sum_{i=1}^{d} \sin \left(x_{i}\right)\left[\sin \left(\frac{i x_{i}}{\pi}\right)\right]^{2 m},(m=10)$,

With $0 \leq x_{i} \leq \Pi$ and $i=1,2,---, d$. The global minima is $\mathrm{f}_{*} \approx-1.801$ for $\mathrm{d}=2$, whereas $\mathrm{f}_{*} \approx-4.6877$ for $\mathrm{d}=5$.

\section{Comparative Study of CS with PSO and GA}

According to the recent research, PSO algorithms left behind GAs and other traditional algorithms for many optimization problems. This is because of the attributes in the broadcasting ability of the current best estimates which promise better and quicker convergence of optimality. It is here been shown the performance of CS versus that of PSO and GAs using most of the standard test functions. The algorithms were run more than hundred times for implementation of Matlab simulation to get meaningful statistical interpretation. The algorithms stop for the variation function values which 
are less than threshold tolerance $\varepsilon \leq 10^{-5}$. The Table 1 shows the results when the global optimas are reached. The format of the table is: average number of evaluations (success rate), i.e., 3221 indicate the average number (mean) of the function evaluation is 3221 with standard deviation 519. The success rate of searching for the global optima for this algorithm is $100 \%$. It is also clear that CS is far more efficient in searching the global optima with the highest $(100 \%)$ success rate. These evaluation processes of each test function are practically instantaneous with a $3 \mathrm{GHz}$ desktop (personal) computer performing 10,000 evaluations in 5 seconds. For all the aforesaid test functions, CS has outperformed both GAs and PSO. The main reasons are: excellent balance between randomisation and intensification with few number of control parameters.

An efficient metaheuristic algorithm is specified by a good balance between intensive local search strategy and an efficient exploration of the whole search space. Moreover, there are only two search parameters in this algorithm; the population size $n$ and $p_{a}$. Once $n$ is fixed, $\mathrm{p}_{\mathrm{a}}$ primarily supervilses the elitism and in turn controls between the randomization and local search. Due to involvement of two parameters the CS algorithm becomes less complex and hence more fundamental. After original CS, there are few new researches that have come out with more complex parameters taking into account. More marvelous results are generated ending up with the aim of speed of convergence.

TABLE I. COMPARISON OF CS WITH GENETIC ALGORITHMS

\begin{tabular}{|c|c|c|c|c|c|c|c|c|c|c|}
\hline $\begin{array}{l}\text { Algor } \\
\text { ithms } \\
\text { /Func } \\
\text { tionss }\end{array}$ & $\begin{array}{l}\text { Multiple } \\
\text { Peaks }\end{array}$ & $\begin{array}{l}\text { Michalew } \\
\text { iez's } \\
(\mathrm{d}=16)\end{array}$ & $\begin{array}{l}\text { Rosenbr } \\
\text { ock's } \\
\text { (d=16) }\end{array}$ & $\begin{array}{l}\text { De } \\
\text { Jong's } \\
(\mathrm{d}=256)\end{array}$ & $\begin{array}{l}\text { Schwefe } \\
\text { l's } \\
(\mathrm{d}=128)\end{array}$ & $\begin{array}{c}\text { Ackley } \\
\text { 's } \\
(\mathrm{d}=128 \\
)\end{array}$ & $\begin{array}{l}\text { Rastrigi } \\
\text { n's }\end{array}$ & Easom's & $\begin{array}{c}\text { Griewan } \\
\text { k's }\end{array}$ & $\begin{array}{l}\text { Shubert } \\
\text { 's }(18 \\
\text { minima) }\end{array}$ \\
\hline GA & $\begin{array}{l}52124 \pm \\
3277 \\
(98 \%)\end{array}$ & $\begin{array}{c}89325 \pm \\
7914 \\
(95 \%)\end{array}$ & $\begin{array}{c}55732 \pm \\
8901 \\
(90 \%)\end{array}$ & $\begin{array}{c}25412 \pm \\
1237 \\
(100 \%)\end{array}$ & $\begin{array}{l}227329 \\
\pm 7572 \\
(95 \%)\end{array}$ & $\begin{array}{l}32720 \pm \\
3327 \\
(90 \%)\end{array}$ & $\begin{array}{l}110523 \\
\pm 5199 \\
(77 \%)\end{array}$ & $\begin{array}{c}19239 \pm \\
3307 \\
(92 \%)\end{array}$ & $\begin{array}{c}70925 \pm \\
7652 \\
(90 \%)\end{array}$ & $\begin{array}{l}54077 \pm \\
4997 \\
(89 \%)\end{array}$ \\
\hline PSO & $\begin{array}{l}3719 \pm \\
205 \\
(97 \%)\end{array}$ & $\begin{array}{c}6922 \pm \\
537 \\
(98 \%)\end{array}$ & $\begin{array}{c}32756 \pm \\
5325 \\
(98 \%)\end{array}$ & $\begin{array}{c}17040 \pm \\
1123(10 \\
0 \%)\end{array}$ & $\begin{array}{l}14522 \pm \\
1275 \\
(97 \%)\end{array}$ & $\begin{array}{l}23407 \pm \\
4325 \\
(92 \%)\end{array}$ & $\begin{array}{c}79491 \pm \\
3715 \\
(90 \%)\end{array}$ & $\begin{array}{c}17273 \pm \\
2929 \\
(90 \%)\end{array}$ & $\begin{array}{c}55970 \pm \\
4223 \\
(92 \%)\end{array}$ & $\begin{array}{l}23992 \pm \\
37557 \\
(92 \%)\end{array}$ \\
\hline CS & $\begin{array}{c}927 \pm \\
105 \\
(100 \%)\end{array}$ & $\begin{array}{c}3221 \pm \\
519 \\
(100 \%)\end{array}$ & $\begin{array}{c}5923 \pm \\
1937 \\
(100 \%)\end{array}$ & $\begin{array}{c}4971 \pm \\
754 \\
(100 \%)\end{array}$ & $\begin{array}{c}8829 \pm \\
625 \\
(100 \%)\end{array}$ & $\begin{array}{c}4936 \pm \\
903 \\
(100 \%)\end{array}$ & $\begin{array}{c}10354 \pm \\
3755 \\
(100 \%)\end{array}$ & $\begin{array}{c}6751 \pm \\
1902 \\
(100 \%)\end{array}$ & $\begin{array}{l}10912 \pm \\
4050 \\
(100 \%)\end{array}$ & $\begin{array}{c}9770 \pm \\
3592 \\
(100 \%)\end{array}$ \\
\hline
\end{tabular}

\section{CONCLUSION}

Authors have studied the CS algorithm which is mainly centered on some species of adult cuckoos' astonishing obligatory brood parasitism along with Levy Flight behavior. Yang and Deb opened up a new avenue by introducing the Lèvy-flight-style rather than the simple random walk. The proposed CS algorithm is validated and compared with some classical Algorithms: GAs and PSO. From simulation and comparison, it is evident that CS is much better and efficient than the GAs and PSO for multimodal objective functions. This is because of the fact that fewer parameters are to be fine controlled in case of CS. It is also clear from the earlier discussion that once $n$, the number of nests, is fixed, only one parameter $\mathrm{p}_{\mathrm{a}}$ is left to be tuned. It should also be noted that the convergence rate in CS using all the aforesaid test functions are very fast and efficient without the interference of $\mathrm{p}_{\mathrm{a}}$. That indicates that these two parameters do not require any fine control for a problem of interest. Consequently CS is more generic and robust for many optimization problems in comparison with other met heuristic algorithms. This promising robust optimization algorithm can effortlessly be adapted to investigate multi-objective optimization approach with various constraints, even to NP-hard problem. More investigations can be carried out with more complex parameters for sensitivity of the optimization and derive any possible relationship out of the problem and the speed of convergence as well as the computational cost. Although CS is a subfield of Swarm Intelligence based algorithms, hybridization can be developed with PSO, GAs and other algorithms which will overcome the drawbacks of CS and will produce robust outcome.

\section{REFERENCES}

[1] Civicioglu. P., Besdok. E., A conceptual comparison of Cuckoo-Search, particle search optimisation, differential evolution and artificial bee colony algorithms, Springer Science and Business Media B. V.2011.

[2] Walton S., Hassan O., Morgan K., and Brown M. R., Modified cuckoo search: A new gradient free optimisation algorithm, Chois, Solutions and Fractals, 44, issue 9, pp 710-718(September 2011).

[3] Barthemy P., Bertolotti J., Wiersma D. S., A Levy Flight for light, Nature, 453, 495-498(2008). 
http://www.google.co.in/imgres?q=levy+distributi on $\&$ hl $=$ en $\&$ sa $=X \&$ tbo $=d \& b i w=1360 \& b i h=629 \& \mathrm{t}$ bm=isch\&tbnid=S2sLMB8a2_KFsM:\&imgrefurl= http://toshiclark.xanga.com/726841489/affirmative -action-redux/\&docid=v5KrG_1jRGKsiM\& imgurl=http://i43.tinypic.com/2216o0.png\&w=496 $\& \mathrm{~h}=393 \&$ ei=zcKpUMupMcSmrAehsoDoAw\&zoo $\mathrm{m}=1$ \&iact=rc\&dur=327\&sig $=1023991376222725$ $30481 \&$ page $=1 \&$ tbnh $=138 \&$ tbnw $=164 \&$ start $=0 \& n$ $\mathrm{dsp}=20 \& v e d=1 \mathrm{t}: 429, \mathrm{r}: 2, \mathrm{~s}: 0, \mathrm{i}: 77 \& \mathrm{tx}=110 \& \mathrm{ty}=68$.

[4] X.-S. Yan, Harmony Search as a Metaheuristic Algorithm, in Music-Inspired Harmony Search Algorithm: Theory and Applications, Studies in Computational Intelligence,Springer Berlin, vol. 191,pp. 1-44(2009).

[5] http://www.metaheuristic.com/x_algorithm_metah euristic_optimization.php.

[6] Bonabeau E., Dorigo M., Theraulaz G., Swarm Intelligance: From Natural to Artificial Systems. Oxford University Press, (1999).

[7] Bulm C. and Roli A., Metaheuristics in combinatorial optimization: Overview and conceptural comparison, ACM Comput. Surv, 35,268-308(2003).

[8] Barthelemy P., Bertelotti J., Wiersma D. S., A Lévy flight for light.Nature, 453, 495-498(2008).

[9] Goldberg D. E., Genetic Algorithms in Search, Optimisation and Mechine Learning, Reading. Mass: Addision Wesley (1989).

[10] Kennedy J. and Eberhart R. C.: Particle swarm optimization.Proc. of IEEE International Conference on Neural Networks. Piscataway, NJ. pp. 1942-1948 (1995).

[11] Yang X. S., Nature-Inspired Metaheuristic Algorithms, Luniver Press, (2008).

[12] Yang X. S., Biology-derived algorithms in engineering optimization (Chapter 32), in Handbook of Bioinspired Algorithms and Applications (eds Olarius \& Zomaya), Chapman \& Hall/ CRC (2005).

[13] Shlesinger M. F., Zaslavsky G. M. and Frisch U. (Eds), Lévy Flights and Related Topics in Physics, Springer, (1995).

[14] Shilane D., Martikainen J., Dudoit S., Ovaska S. J., A general framework for statistical performance comparison of evolutionary computation algorithms, Informational Sciences: an Int. Journal, 178,2870-2879(2008).

[15] Shang Y. W., Qiu Y. H., A note on the extended rosenrbock function, Evolutionary Computation, 14,119-126(2006).

[16] Schoen F., A wide class of test functions for global optimization, J. Global Optimization, 3,133-137, (1993).

[17] Kennedy J., Eberhart R., Shi Y.: Swarm intelligence, Academic Press, (2001).

[18] Payne R. B., Sorenson M. D., and Kiltz K., The Cuckoos, Oxford University Press, (2005).

[19] Reynolds A. M. and Frye M. A. ,Free-flight odor tracking in Drosophilia is consistent with an optimal intermittent scale-free search, PLoS One,2,e354(2007).

[20] Xin-She Yang,Suash Deb,Cuckoo Search via Le'vy Flights. Proc. Of World Congress on Nature \& Biologically Inspired Computing (NaBIC 2009), India. IEEE Publications, USA.

[21] Shlesinger M. F, Search Research, Nature, 443,281- 282(2006).

Sangita Roy is an Assistant Professor at ECE Department of Narula Institute of Technology under WBUT .She has a teaching experience of more than sixteen years. She was in instrumentation industry for two years and in administration for two years. She completed her Diploma (ETCE), A.M.I.E (ECE) and MTech (Comm. Engg).Currently perusing her PhD under Dr. Sheli Sinha Chaudhuri at ETCE Department of Jadavpur University. She is a member of IEI, IETE, FOSET, and IEEE.

Dr. Sheli Sinha Chaudhuri is an Associate Professor at ETCE Department of Jaduvpur University. She completed her B-Tech, M-Tech, and $\mathrm{PhD}$ at Jadavpur University. She has a vast teaching experience of 14 years. She has large number of papers in International and national level journals as well as conferences. Currently research scholars are pursuing $\mathrm{PhD}$ under her guidance. She is a member of IEEE and IEI. 